\title{
Early Detection of Problem Gambling based on Behavioral Changes using Shapelets
}

\author{
Hiroko Suzuki \\ Dept. of Computer Science, \\ Meiji University \\ Fujitsu Laboratories Ltd. \\ Kanagawa, Japan \\ hiroko.suzuki@fujitsu.com
}

Isamu Watanabe

Fujitsu Laboratories Ltd.

Kanagawa, Japan

watanabe.isamu@fujitsu.com

\author{
Ryoko Nakamura \\ Dept. of Computer Science, \\ Meiji University \\ Kanagawa, Japan \\ ry5983rsg@cs.meiji.ac.jp
}

\author{
Aozora Inagaki \\ Dept. of Computer Science, \\ Meiji University \\ Kanagawa, Japan \\ bluezora@cs.meiji.ac.jp
}

\author{
Tomohiro Takagi \\ Dept. of Computer Science, \\ Meiji University \\ Kanagawa, Japan \\ takagi@cs.meiji.ac.jp
}

\begin{abstract}
Recent years have seen strides achieved in the field of behavior analysis by using online gambling data. However, studies on time-series behavioral changes remain inadequate. In this study, we propose a classifier that quantifies changes in the player's time series of online gambling behavioral data by using distance measurement with shapelet for the early detection of behaviors in players that could lead to problem gambling. We investigated the prediction capabilities of shapelets that represent behavioral change patterns, and the results showed that shapelet features can improve predictive accuracy. Furthermore, based on this result, we found characteristic behavioral changes leading to problem gambling, such as loss chasing. Subsequently, we demonstrated a possibility for improvements in accuracy using these behavioral change patterns based on expert knowledge.
\end{abstract}

\section{CCS CONCEPTS}

-Information systems $\rightarrow$ Data mining; • Applied computing $\rightarrow$ Computer games.

\section{KEYWORDS}

Shapelet, Behavioral Feature Extraction, Early Detection, Problem Gambling, Online Gambling

\section{ACM Reference Format:}

Hiroko Suzuki, Ryoko Nakamura, Aozora Inagaki, Isamu Watanabe, and Tomohiro Takagi. 2019. Early Detection of Problem Gambling based on Behavioral Changes using Shapelets. In IEEE/WIC/ACM International Conference on Web Intelligence (WI '19), October 14-17, 2019, Thessaloniki, Greece. ACM, New York, NY, USA, 6 pages. https://doi.org/10.1145/3350546.3352549

Permission to make digital or hard copies of all or part of this work for personal or classroom use is granted without fee provided that copies are not made or distributed for profit or commercial advantage and that copies bear this notice and the full citation on the first page. Copyrights for components of this work owned by others than ACM must be honored. Abstracting with credit is permitted. To copy otherwise, or republish, to post on servers or to redistribute to lists, requires prior specific permission and/or a fee. Request permissions from permissions@acm.org.

WI '19, October 14-17, 2019, Thessaloniki, Greece

(c) 2019 Association for Computing Machinery.

ACM ISBN 978-1-4503-6934-3/19/10 . \$ \$15.00

https://doi.org/10.1145/3350546.3352549

\section{INTRODUCTION}

In recent years, the need for research on the early detection and prevention of gambling addiction has increased as the discussion on the opening of casinos in Japan has progressed.

In particular, studies in the field of behavior analysis, using online gambling data, have focused on the early detection of highrisk players for "problem gambling" (a situation in which gambling negatively affects the players' life, hereinafter referred to as PG). For the early detection of players heading towards PG, it is important to consider not only static gambling behavioral characteristics but dynamic and temporal change of gambling behavior.

For example, in the field of gambling addiction medicine, it is known that specific behaviors such as "loss chasing," in which the player attempts to recover losses by increasing the number of bets after experiencing a big loss, is observed [2]. Because players partially exhibit such characteristic behaviors leading to PG throughout their career, simple statistical values such as the summary values and the average of the entire career are insufficient. In addition, it is difficult to quantify behavioral change because the duration of a gambling career (the number of days since the player plays for the first time) varies from player to player and their value fluctuates irregularly depending on the frequency of visits, among other factors. Hence, this not been studied extensively. In this research, we conducted the following two analyses:

(Analysis 1) We propose a method to quantify player's behavioral change using shapelets that represents the temporal change in time-series data. The shapelets are used as features to predict if the players will lead to PG. In addition, the effectiveness of the proposed method is compared with that of the baseline method, conducted in prior research, which predicts high-risk players using only features such as basic behavioral characteristics and demographic attributes.

(Analysis 2) Furthermore, based on this result, we examine whether characteristic behavioral changes that lead to PGs, such as loss chasing, can be extracted and confirm if there is a possibility of improvement in accuracy using expert knowledge.

The remainder of the paper is structured as follows. In Section 2, we introduce related research to form the baseline of this study. In 
Section 3, we explain data processing flow as an overview of our proposed method. The details of the construction of shapelet features, which is the essential part of our method, are also described. In Section 4, we elaborate upon the dataset used in this study. In Section 5, we explain the results of two analyses conducted in this study. In Section 6, we provide the conclusions.

\section{RELATED RESEARCH}

Research that focused on behavior analysis using online gambling data in the past 10 years was investigated [5]. In most cases, the analysis is performed using the total number of plays in the total period, basic behavior features (total bet amount), and demographic attribute features (gender and age) [7],[4]. Therefore, analysis using temporal behavioral change in a player leading to PG remains lacking.

For extracting a characteristic time series during classification, there exists a method for extracting shapelets that minimizes the loss function of class classification from multiple shapelets (timeseries feature patterns common to classes) [6]. In this study, we used the previously proposed shapelets generation method implemented in the "tslearn" library (Python).

In addition, to design a method for generating time-series feature data using shapelets for classification, studies were conducted using similarity obtained as time-series feature data by calculating the similarity between shapelets and time-series data using Euclidean distance (ED), such as [8] and their application to classification of time-dependent multivariate time series [3]. For the distance measurement of time-series data, ED is effective for measuring the similarity in shape and the dynamic time warping (DTW) method is effective for measuring similarity in the shape [1].

Therefore, in this research, we use the characteristics of both ED and DTW to generate time-series features effective for time-series classification. We use ED to identify the time at which the feature pattern (shapelets) appears in the classification. To determine the value and time width for behaviors that are player dependent, such as wagering, DTW is used as it provides a more detailed measurement.

\section{PROPOSED METHOD}

\subsection{Overview of proposed method}

Figure 1 shows the processing flow of the proposed method in this research. According to the general machine learning framework, the data set is divided into training and test data sets, and feature extraction is performed on each of them. A binary classification model to identify PG is created from a training data set using a classifier such as logistic regression; the prediction result is output by applying this model to a test data set.

The flow of feature amount creation is shown in Figure 2. First, basic behavioral and demographic features are generated from the data with reference to previous research; these features are then combined with features generated from shapelets.

\subsection{Construction of shapelet features}

The overall flow of feature extraction is shown in Figure 3.

(a) Shapelet Extraction: Data set $D$ includes multidimensional timeseries data such as number of bets, Hold, Turnover, and correct
Training Phase

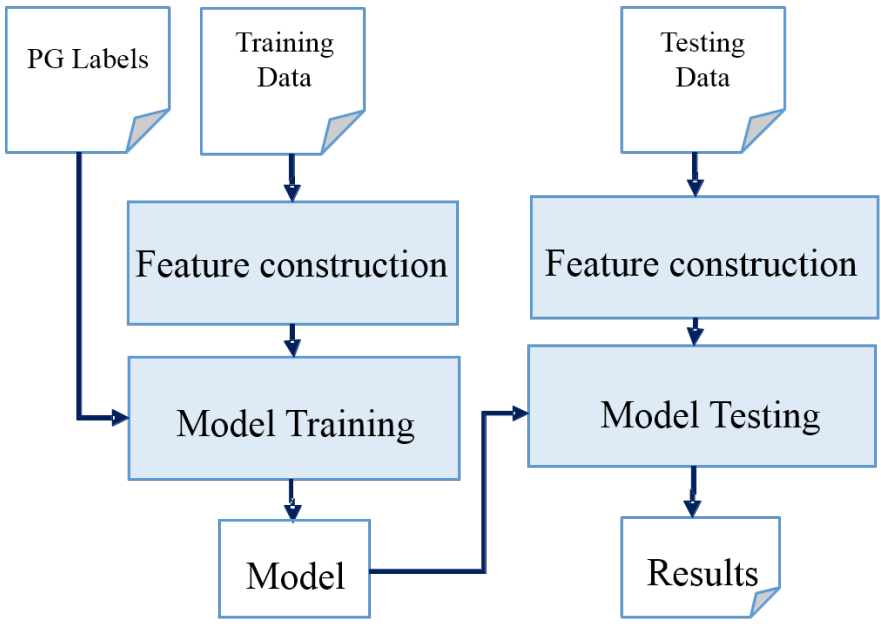

Figure 1: Overview (Training and Testing)

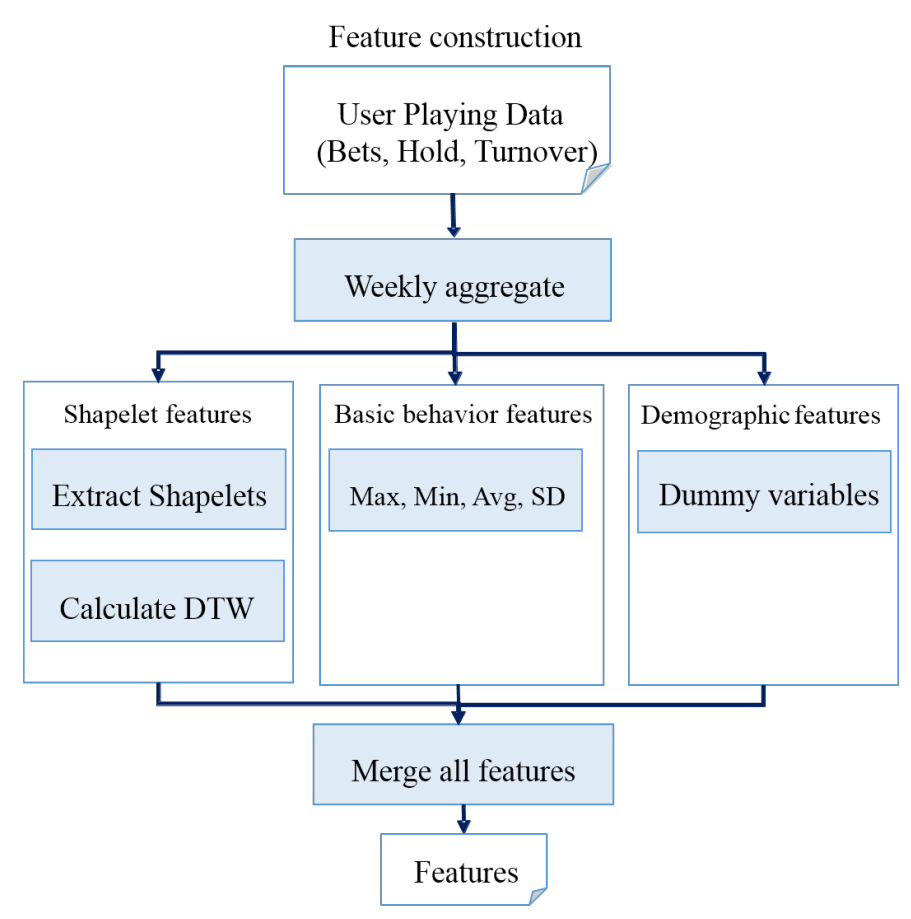

Figure 2: Flow of feature construction

answer labels. When the player reaches PG, the correct answer label is 1 ; else, it is 0 . A set of data of players (length $=n$ ) is denoted as $X=\left\{x_{i}, i=1, \ldots, n\right\}$, a set of PG labels $y_{i}=\{0,1\}$ is denoted as $Y=\left\{y_{i}, i=1, \ldots, n\right\}$, a set of time-series data of $L$ of each user is defined as $M T=\left\{m t_{i}, i=1, \ldots, n\right\}$. Further, a set of the entire data is $X$, and it is divided into training and test data, which are represented as $X$ train and $X$ test, respectively. The PG labels that 
(a) Shapelet extraction

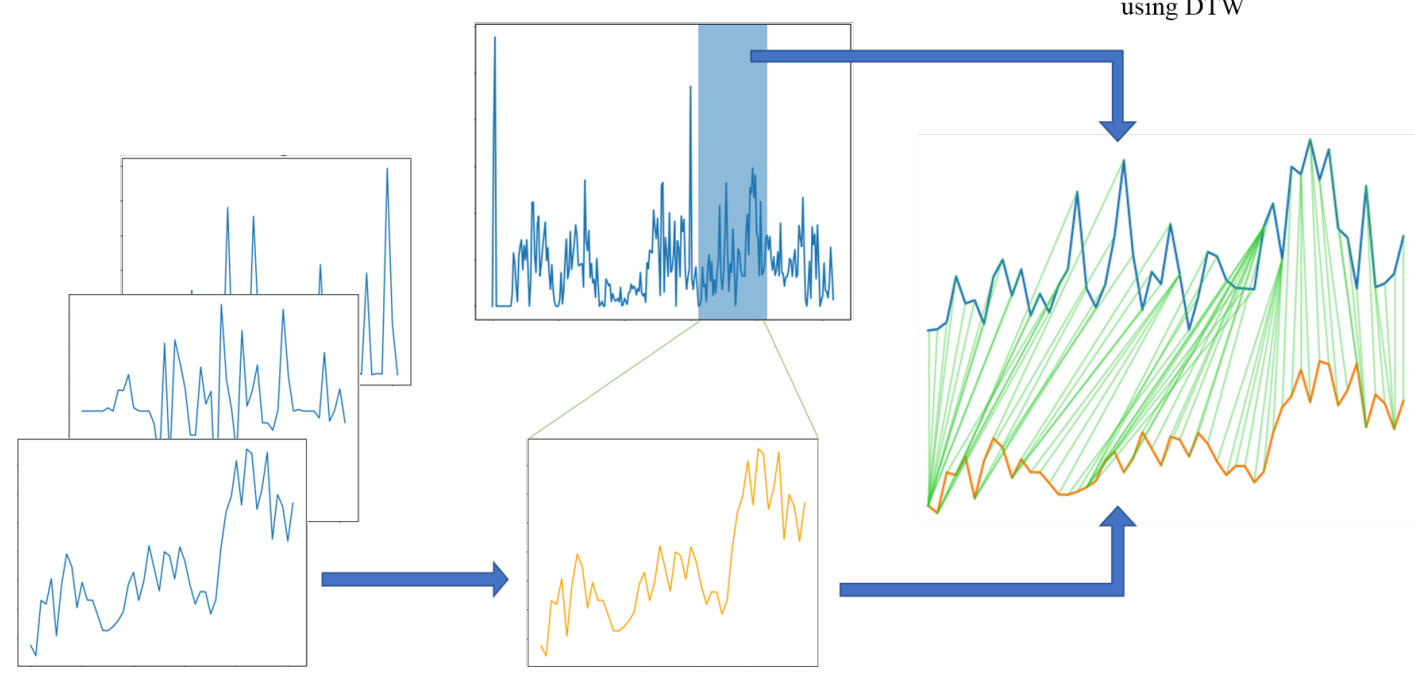

(c) Calculation of exact similarity using DTW

Figure 3: Steps of similarity calculations

correspond to training data and test data are shown as Ytrain and Ytest, respectively.

Shapelets are time-series feature patterns common to the same class, and here, we extract shapelets from the training data $X$ train. Furthermore, classification is performed internally with the distance between shapelets and time series data as the feature, and the shapelets are learned to minimize the loss function [6]. This allows the selection of shapelets that are valid for classification. In the case of classification in PG, it is possible to extract shapelets that show effective behavioral changes in the classification of players to PG. Thus, a set of these extracted shapelets is denoted as $S=$ $\left\{s_{k, l}, k=1, \ldots, p\right\}$, where $p$ denotes the number of pieces of the $k$-th element of length $l$. The length $l$ and the number $p$ of shapelets need to be specified manually or by the method that determines the optimum parameter.

(b) Matching using ED: To explore the timing at which a timeseries change appears to be similar to that in an extracted shapelet, we calculate the ED between the local time series created by a sliding window and that created by each shapelet. In particular, the time-series data $m t_{i}$ for each player $i$ is divided into the time interval $[t, t+l]$, for which the length is $l$ (initial value is $t=0$ ), and this local time series is $w_{t, l}$. Then, we calculate the ED distance $D_{\mathrm{ED}}\left(s_{k, l}, w_{t, l}\right)$ between this $w_{t, l}$ and each shapelet $s_{k, l}$. Next, we increment $t$ by 1 , which is the starting point of the time interval; we then repeatedly calculate the ED distance with a new local time series $w_{t, l}$ by using the sliding window method. Finally, we find the local time series $w_{t, l}^{\prime}$ that minimizes the distance.

$$
w_{t, l}^{\prime}=\underset{w_{t, l} \in m t_{i}}{\arg \min } D_{\mathrm{ED}}\left(s_{k, l}, w_{t, l}\right)
$$

(c) Calculation of exact similarity using DTW: To measure the distance accurately, the DTW distance $D_{\mathrm{DTW}}\left(s_{k, l}, w_{t, l}^{\prime}\right)$ is calculated using the DTW that can be expanded and contracted in time; this value $D_{\mathrm{DTW}}\left(s_{k, l}, w_{t, l}^{\prime}\right)$ is the feature corresponding to each shapelet of player $i$. This process is determined for all $p$ shapelets in an $m$-dimensional time series, and finally, $m \times p$ features are obtained.

\section{DATASET}

This study uses the dataset ${ }^{1}$ used in [4]. An online gambling vendor that provided the dataset developed procedures to identify PG events for responsible gambling intervention. PG events includes "account closure due to PG," "request to raise personal deposit limit," and so on. This dataset contains gambling behavioral information of 2,068 players as positive cases (players who have experienced PG events); the dataset also contains 2,066 players as negative cases (players who have not experienced PG events). RawDataset1 contains the demographic attribute information for a player, and the PG labels of players who joined online gambling during the period from September 17, 1999 to November 27, 2009; RawDataset2 contains the daily playing $\log$ of the player from May 1, 2000 to November 10, 2010; and RawDataset3 contains the details of the PG events.

In this study, we used the data of 4,113 players (2,068 PG players and 2,045 non-PG players); we exclude 21 players from this data set as their records contain missing values. In particular, we used gender and year of birth as demographic attribute information from RawDataset1 and the amount of bets (Turnover), amount of loss (Hold), and number of bets from RawDataset 2. As a part of preprocessing, the daily playing log was aggregated weekly, and for periods when there was no play, the data was interpolated to 0 .

\footnotetext{
${ }^{1}$ Division on Addiction, Behavioral characteristics of Internet gamblers who trigger corporate responsible gambling interventions.Medford, MA: Division on Addiction, The Transparency Project [database distributor], February 7, 2016.
} 
Table 1: Details of features

\begin{tabular}{cccccc}
\hline Category & items & Explanation & \#of variables & Baseline & Proposed method \\
\hline Demographic features & Gender & Dummy variables (0,1) & 1 & $\checkmark$ & $\checkmark$ \\
\hline & Age & Categorical variables (10s, 20s, ..) & 1 & $\checkmark$ & $\checkmark$ \\
\hline Basic behavior features & Turnover & Max, Min, Average, SD & 4 & $\checkmark$ & $\times$ \\
\hline & \#Bets & Max, Min, Average, SD & 4 & $\checkmark$ & $\times$ \\
\hline & Hold & Max, Min, Average, SD & 4 & $\checkmark$ & $\times$ \\
\hline Shapelets features & Turnover & Similarity with Shapelets & 6 & $\times$ & $\checkmark$ \\
\hline & \#Bets & Similarity with Shapelets & 6 & $\times$ & $\checkmark$ \\
\hline \multicolumn{7}{c}{ Told } & Similarity with Shapelets & 6 & $\times$ & 14 \\
\hline
\end{tabular}

\section{ANALYSIS}

As introduced in Section 1, we conducted the following two analyses. In analysis 1 , we verified whether the classification accuracy of the player improves using features generated from shapelets. In analysis 2 , based on the results of analysis 1 , we examined characteristic behavioral changes patterns leading to PG and confirmed whether these patterns would improve the prediction accuracy for PG players.

\subsection{Analysis 1: Prediction of PG players using shapelets}

The features used in the baseline and the proposed method are listed in Table 1. Players have a three-dimensional weekly time-series data: total amount of bets (Turnover), total amount of loss (Hold), and the total number of bets. From these three time-series data, a basic behavior feature and other shapelets features are generated. A baseline uses a total of 14 features that comprise demographic attribute and basic behavior features. On the contrary, the proposed method uses 20 features that comprise demographic attribute and shapelet features. In particular, 18 shapelets of length 52 (weeks) were extracted from the three time-series data and this similarity was used as the feature.

Two examples of extracted shapelets are shown in Figure 4. The figure on the left is a shapelet of the transition of weekly losses (Hold). The $\mathrm{X}$ axis is a relative week where the origin of the extracted shapelet is zero. The y-axis shows the loss amount in euros. This shapelet shows loss during the first five weeks and repeated big losses and big wins. The figure on the right is the shapelet for the weekly betting trend. The y-axis shows the number of bets. Irrespective of small up and down patterns, the overall betting frequency gradually increases.

In addition, because there are more variables than the baseline and the features generated by shapelet have high correlation with each other, one of the pair of variables with high correlation coefficient ( 0.9 or more) is manually removed. A total of 15 variables were obtained. The evaluation results of each method are summarized in Table 2. The precision was the highest for the baseline method; however, accuracy, recall, and F1 were higher for the proposed method, in particular, for the proposed method and a selected variable.
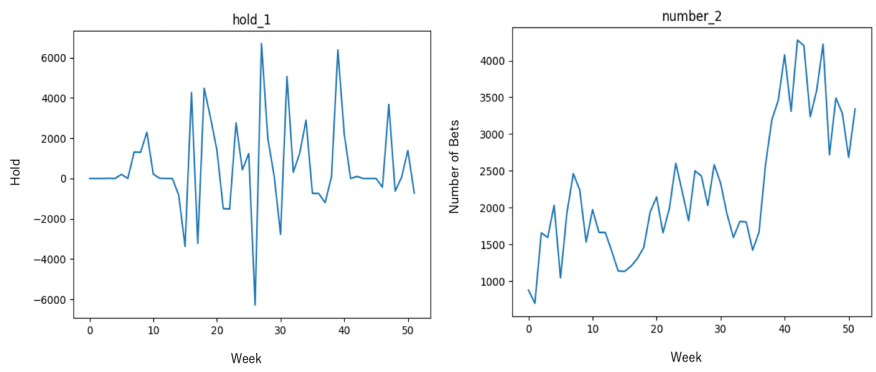

Figure 4: Extracted Shapelets

Table 2: Evaluation Results for Analysis 1

\begin{tabular}{cccc}
\hline & Baseline & Proposed & $\begin{array}{c}\text { Proposed } \\
\text { (feature selection) }\end{array}$ \\
\hline Accuracy & 0.777 & 0.790 & 0.793 \\
\hline Precision & 0.869 & 0.825 & 0.827 \\
\hline Recall & 0.655 & 0.740 & 0.745 \\
\hline F1 & 0.747 & 0.780 & 0.784 \\
\hline
\end{tabular}

\subsection{Analysis 2: Identify the position of behavioral changes}

From Analysis 1, it is clear that the feature values generated by shapelet are effective in PG classification. In Analysis 2, the period with the highest similarity with the extracted shapelet is visualized in the entire period of the actual player, and the actual behavioral change captured by the shapelets are confirmed. Figure 5 shows two obtained PG player cases. The upper row shows the change in weekly losses (Hold expressed in euros), and the lower row shows the change in the weekly numbers of bets. The change in weekly amount of bets (Turnover) is omitted in the figure because it is almost the same as the change in the number of bets. The $\mathrm{X}$ axis indicates the number of weeks since the week after the first play. The 0 on the left end indicates the start of play, and the right end indicates the timing of the occurrence of the PG event.

The first player had been placing a relatively moderate number of bets (about 150) from the beginning, and the loss amount was almost 


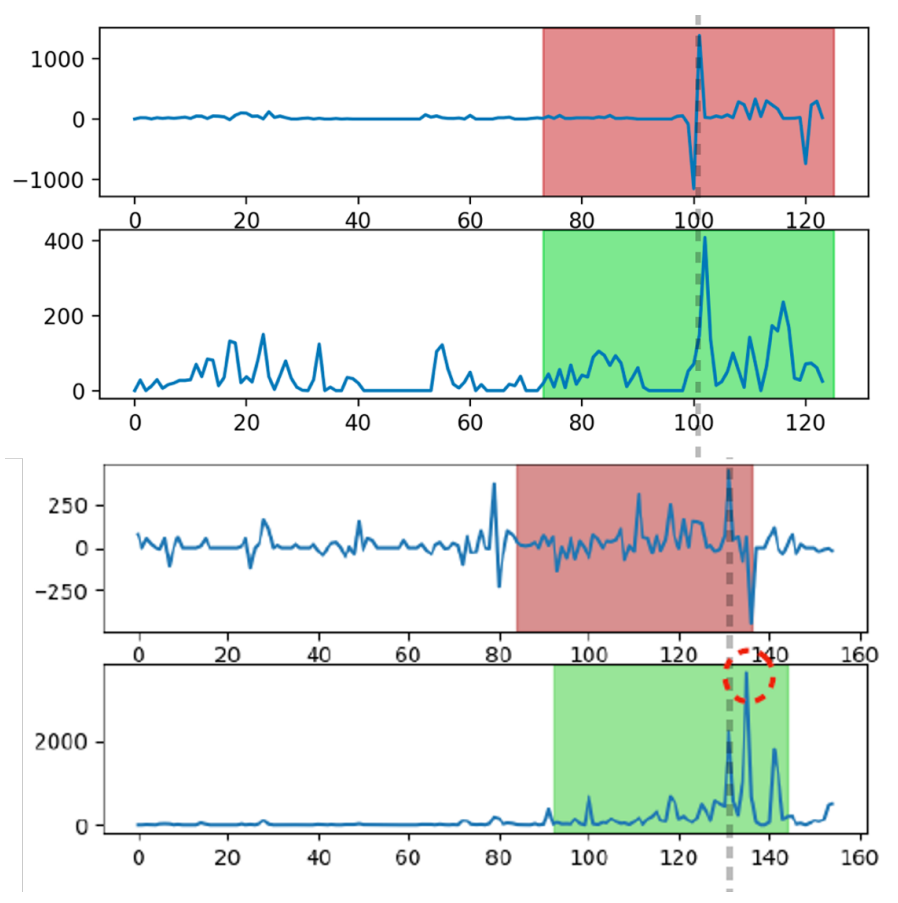

Figure 5: Examples of the PG players

zero, which means that the loss did not exceed the amount of bets. Then, after approximately 100 weeks (around 2 years); the player won approximately 1,000 euros, and the number of bets increased soon after. The player experienced a loss of approximately 1,200 euros (indicated by the dashed line) in the 101st week. Immediately after that, the player placed bets approximately 400 times in the next week, which is more than twice the number of bets placed initially. This can be interpreted as an action to recover from the loss.

Further, the second player had been placing a small number of continual bets from the beginning; however, they performed a large number, approximately 2,000 bets, after about 130 weeks (around 2.5 years). Owing to this, they experienced a loss of more than approximately 250 euros in the 131st week. Then, as of the 135th week, the player made the largest number of bets, approximately 3,000 , so far.

The period in which these characteristic behavioral changes occurred is specified as the point at which the similarity of the shapelet is the highest. (Red and green period). Therefore, it was confirmed that the features generated from the shapelets could capture important behavioral changes and these improve the accuracy of the classification. However, in the proposed method, behavioral changes were extracted independently. Therefore, complex patterns such as loss chasing, which were defined as a combination of a set of shapelets, were not extracted. Thus, there is a possibility that the accuracy can be improved by manually generating such complex features and adding them to the prediction model.

As a supplementary analysis, some patterns of features that express loss chasing are created and added to the proposed method (with variable selected), which is the highest accuracy in Analysis
1. The three following patterns of loss chasing (LC) were created as features to express loss chasing.

- Maximum increase in bets after first loss (+ LC-Max)

- Average of increase in bets after first loss (+ LC-Avg)

- Cumulative sum of increase in bets after first loss (+ LC-Cum)

The evaluation results of each method are shown in Table 3 . The accuracy increased slightly for all three patterns. Additionally, among the three patterns, the patterns that include cumulative sum (+ LCCum) had the highest accuracy, although there is a slight difference. Moreover, when the significance of the added variable was confirmed in the logistic regression, $\mathrm{P}$-value became less than 0.05 , and as a result, it contributed to statistically significant classification.

Table 3: Evaluation Results for Analysis 2

\begin{tabular}{ccccc}
\hline & $\begin{array}{c}\text { Proposed } \\
\text { (feature selection) }\end{array}$ & $\begin{array}{c}\text { +LC } \\
\text {-Max }\end{array}$ & $\begin{array}{c}\text { +LC } \\
\text {-Avg }\end{array}$ & $\begin{array}{c}\text { +LC } \\
\text {-Cum }\end{array}$ \\
\hline Accuracy & 0.793 & 0.794 & 0.796 & 0.798 \\
\hline Precision & 0.827 & 0.825 & 0.826 & 0.829 \\
\hline Recall & 0.745 & 0.749 & 0.753 & 0.753 \\
\hline F1 & 0.784 & 0.785 & 0.788 & 0.789 \\
\hline
\end{tabular}




\section{CONCLUSION}

In this study, we used online gambling data to quantify time-series behavioral changes using shapelet. From prediction experiments on whether players acquired gambling problems using these features, it has become clear that time-series features are more effective than basic behavior features. In addition, when we performed visualization analysis on the location where the degree of similarity with shapelet is the largest, it became evident that the characteristic change in the behavior of PG such as loss chasing was caught to some extent. We also show that there is room for improvement in accuracy by explicitly adding complex features according to the hypothesis in the field. In future, we would like to generate complex features that are defined as a set of shapelets, not manually but automatically. Additionally, we would like to verify the effectiveness of the generation methods for time-series features other than shapelets. We will also consider applying the proposed method to fields such as customer behavior prediction.

\section{REFERENCES}

[1] Sr Aghabozorgi, Teh Wah, Tutut Herawan, Hamid Jalab, Mohammad Shayegan, and Alireza Jalali. 2014. A Hybrid Algorithm for Clustering of Time Series Data Based on Affinity Search Technique. The Scientific World fournal 2014 (2014), 562194.

[2] Alex Blaszczynski and Lia Nower. 2002. A pathways model of problem and pathological gambling. Addiction 97, 5 (2002), 487-499.

[3] Aaron Bostrom and Anthony J. Bagnall. 2017. A Shapelet Transform for Multivariate Time Series Classification. CoRR abs/1712.06428 (2017). arXiv:1712.06428 http://arxiv.org/abs/1712.06428

[4] Julia Braverman, Debi A LaPlante, Sarah E Nelson, and Howard J Shaffer. 2013. Using cross-game behavioral markers for early identification of high-risk internet gamblers. Psychology of Addictive Behaviors 27, 3 (2013), 868.

[5] Bernardo T Chagas and Jorge FS Gomes. 2017. Internet gambling: A critical review of behavioural tracking research. fournal of Gambling Issues 36 (2017).

[6] Josif Grabocka, Nicolas Schilling, Martin Wistuba, and Lars Schmidt-Thieme. 2014. Learning time-series shapelets. In Proceedings of the 20th ACM SIGKDD international conference on Knowledge discovery and data mining. ACM, 392-401.

[7] Heather M Gray, Debi A LaPlante, and Howard J Shaffer. 2012. Behavioral characteristics of Internet gamblers who trigger corporate responsible gambling interventions. Psychology of Addictive Behaviors 26, 3 (2012), 527-535.

[8] Jason Lines, Luke M Davis, Jon Hills, and Anthony Bagnall. 2012. A shapelet transform for time series classification. In Proceedings of the 18th ACM SIGKDD international conference on Knowledge discovery and data mining. ACM, 289-297. 